\title{
BMJ Open The use of the environmental scan in health services delivery research: a scoping review protocol
}

\author{
Patricia Charlton, ${ }^{1}{ }^{1}$ Shelley Doucet, ${ }^{2}$ Rima Azar, ${ }^{3}$ Daniel A Nagel, ${ }^{4}$ Leah Boulos, ${ }^{5}$ \\ Alison Luke, ${ }^{6}$ Kim Mears, ${ }^{7}$ Katherine J Kelly, ${ }^{8}$ William J Montelpare ${ }^{9}$
}

To cite: Charlton P, Doucet S, Azar $\mathrm{R}$, et al. The use of the environmental scan in health services delivery research: a scoping review protocol. BMJ Open 2019;9:e029805. doi:10.1136/ bmjopen-2019-029805

- Prepublication history for this paper is available online. To view these files, please visit the journal online (http://dx.doi org/10.1136/bmjopen-2019029805).

Received 12 February 2019 Revised 10 May 2019 Accepted 25 June 2019

Check for updates

(c) Author(s) (or their employer(s)) 2019. Re-use permitted under CC BY-NC. No commercial re-use. See rights and permissions. Published by BMJ.

For numbered affiliations see end of article.

Correspondence to Dr Patricia Charlton; pcharlton@upei.ca

\section{ABSTRACT}

Introduction The environmental scan has been described as an important tool to inform decision-making on policy, planning and programme development in the healthcare sector. Despite the wide adoption of environmental scans, there is no consensus on a working definition within the health services delivery context and methodological guidance on the design and implementation of this approach is lacking in the literature. The objectives of this study are to map the extent, range and nature of evidence that describe the definitions, characteristics, conceptualisations, theoretical underpinnings, study limitations and other features of the environmental scan in the health services delivery literature and to propose a working definition specific to this context.

Methods and analysis This protocol describes a scoping review based on the methodology outlined by Khalil and colleagues. A comprehensive search strategy was developed by experienced health science librarians in consultation with the research team. A Peer Review of Electronic Search Strategies (PRESS) was completed. Two reviewers will independently screen titles, abstracts and full-text articles and select studies meeting the inclusion criteria from seven electronic databases: Academic Search Premier, Canadian Business \& Current Affairs (CBCA), CINAHL, ERIC, Embase, MEDLINE and PsycINFO. The grey literature and reference lists of included articles will also be searched. The data will be analysed and presented in tabular format, and will include a descriptive numerical summary as well as a qualitative thematic analysis. Ethics and dissemination This protocol provides an audit trail for a scoping review that will advance understanding about the environmental scan and its application in the health services delivery context. The review will propose a working definition and will inform future research to explore the development of a conceptual framework in this context. Findings will be disseminated through a peer-reviewed journal and conference presentations. The scoping review does not require ethics approval.

\section{BACKGROUND}

\section{Purpose of environmental scanning}

Emanating from the business worlds over the past half century, environmental scanning entails the process of seeking, gathering, interpreting and using information from the internal and external environments

\section{Strengths and limitations of this study}

This paper describes the scoping review protocol that we will follow to systematically examine the application of environmental scans within the health services delivery context, an area of research that has not been comprehensively reviewed.

- In consultation with the research team, experienced health sciences librarians developed the comprehensive three-step search strategy according to established scoping review methodology.

- The inclusion criteria include both peer-reviewed and grey literature to ensure comprehensiveness but will be limited to publications in French and English.

- The studies included in the scoping review will not be assessed for methodological quality.

- The breadth of sources may result in a large, unmanageable volume of references which may require refinement to the inclusion criteria, and relevant reports or studies may be missed if they are not available in the public or scientific domain.

of an organisation to inform strategic decision-making and to direct future organisational action. ${ }^{1-5}$ Environmental scanning enables organisations to identify, assess and understand elements in the environment that may be perceived as potential threats or opportunities, and respond to developing issues that could impact operations, corporate success and sustainability. ${ }^{4-7}$ The process can entail an analysis of the technological, regulatory, economic, social, cultural, linguistic, geographical or political environments. ${ }^{6-9}$

Scanning the environment is integral to strategic planning and is linked to improved organisational performance. ${ }^{4}{ }^{6-12}$ Organisations can take a reactive or proactive approach to scanning, and the frequency of scanning activity can range from irregular (eg, for a particular purpose such as crisis response) to more advanced continuous scanning (eg, broad monitoring and analysis to support planning and decision-making). ${ }^{2}$ Scanning 
is more frequent when organisations have higher levels of perceived uncertainty. ${ }^{4-61314}$ Perceived uncertainty has been interpreted as the rate of change in the environment (ie, dynamism) and the number of factors or elements in the environment (ie, complexity) that are important to the organisation and their decision-making and that can potentially impact performance. ${ }^{14-17}$

\section{Environmental scanning and the healthcare sector}

Environmental scanning is particularly relevant for informing decision-making and strategic planning in the healthcare sector, ${ }^{10-12}{ }^{18-23}$ which is commonly characterised as a complex, dynamic and turbulent environment. ${ }^{24-28}$ The environmental scan has been described as an important and effective public health tool to inform policy, planning and programme development. ${ }^{12} 29-31$ Identifying potential threats, opportunities and emerging critical issues in the social, political, regulatory, technological and economic environments is integral to planning and can help organisations foresee, understand, prepare for and address the pressures, trends and issues facing healthcare. ${ }^{11} 1619212332-37$ These pressures include rising rates of chronic disease, changing demographics, increasing consumer expectations, growing service demands, service quality issues, human resources challenges and financial constraints. ${ }^{16} 19232831$ 34-37

Environmental scans have been used as an effective approach to information gathering for a range of specific purposes (table 1). These include reviewing the current state of services and programmes, evaluating community and patient needs, identifying service gaps, assessing professional education and training needs, supporting quality improvement initiatives, and informing programme and policy development. ${ }^{12} 31$ 37-59 For instance, environmental scans have been used to assess remote symptom support training programmes for nurses in ambulatory oncology programmes ${ }^{56}$ and to describe paediatric navigation models across Canada. ${ }^{39}$

\section{Methods, definitions and conceptual models}

Data collection can take a passive or active approach, and scanning modes can include personal, impersonal, internal and/or external sources. ${ }^{412} 2960$ Data sources are wide ranging and can include administrative data, internal reports, clinical guidelines, journals and key informants, and data can be collected through various methods including interviews, observation, surveys and internet searches. ${ }^{48} 1229304552$

Despite the wide adoption of environmental scans within the healthcare sector, ${ }^{12137-59}$ there is no consensus on a working definition or conceptual framework specific to health services delivery, and methodological guidance on the design and implementation of this approach to guide research and practice is lacking in the literature. ${ }^{129-3145}$ Various terminologies have been used to describe the concept. For example, environmental scans have been referred to as a 'needs assessment tool', ${ }^{12}$ 'community health research tool', ${ }^{30}$ 'approach', 'mixed methods
Table 1 Studies and reports utilising environmental scans in the healthcare context

\begin{tabular}{|c|c|}
\hline Purpose & Studies \\
\hline $\begin{array}{l}\text { Examine the current } \\
\text { state of programmes } \\
\text { and services for } \\
\text { specific populations }\end{array}$ & $\begin{array}{l}\text { Association of Maternal Child } \\
\text { Health Programs }{ }^{37} \\
\text { DaCosta et }\left.a\right|^{38} \\
\text { Luke et } a l^{39} \\
\text { Mew et } a l^{40} \\
\text { Rac et al }{ }^{41} \\
\text { Wijeysundera et al }{ }^{42}\end{array}$ \\
\hline $\begin{array}{l}\text { Identify strengths, } \\
\text { challenges and service } \\
\text { gaps }\end{array}$ & $\begin{array}{l}\text { Canadian Mental Health } \\
\text { Association }{ }^{43} \\
\text { DaCosta et a/ } \\
\text { Moore et al }{ }^{44} \\
\text { Naumann et al }{ }^{45}\end{array}$ \\
\hline $\begin{array}{l}\text { Assess community and } \\
\text { patient needs }\end{array}$ & $\begin{array}{l}\text { Gustafson et al }{ }^{46} \\
\text { Porterfield et al } \\
\text { Rowel et al }\end{array}$ \\
\hline $\begin{array}{l}\text { Guide quality } \\
\text { improvement initiatives }\end{array}$ & $\begin{array}{l}\text { Aslakson et } a l^{48} \\
\text { Bednar et } a l^{49} \\
\text { Leas et } a l^{50} \\
\text { Sibbald et } a l^{51}\end{array}$ \\
\hline $\begin{array}{l}\text { Support clinical } \\
\text { practice and } \\
\text { professional education }\end{array}$ & $\begin{array}{l}\text { Hatch and Pearson } \\
\text { Hodges et } a l^{53} \\
\text { Maclean } \\
\text { McPherson et } a l^{55} \\
\text { Stacey et a }\left.\right|^{56}\end{array}$ \\
\hline $\begin{array}{l}\text { Inform healthcare } \\
\text { decision-making } \\
\text { for programme } \\
\text { planning and policy } \\
\text { development }\end{array}$ & $\begin{array}{l}\text { Association of Maternal and Child } \\
\text { Health Programs } \\
\text { Baezconde-Garbanati et }\left.a\right|^{57} \\
\text { Blasi et }\left.a\right|^{58} \\
\text { Jamieson } \\
\text { Wilburn et }\left.a\right|^{31}\end{array}$ \\
\hline
\end{tabular}

approach', 'methodological approach ${ }^{61}$ and 'systematic survey'. ${ }^{62}$

Although several process models or conceptual frameworks for environmental scanning have been developed within the business and education sectors, ${ }^{2-57861}$ few have been developed specifically to guide research and practice in the design, implementation or evaluation of this methodological approach in the health services delivery context. ${ }^{123145}$ Rowel et al ${ }^{12}$ conducted an environmental scan to inform the development and implementation of a cancer screening project. They concluded that the environmental scan can be an important 'tool' to guide planning and project development but suggested that it lacks definition, and recommended more application and evaluation to enhance the research methodology. Furthermore, they proposed that future research could focus on the development of a conceptual model for public health based on the $\mathrm{Choo}^{4}$ model that was developed within the business context. Building on previous frameworks, ${ }^{163}$ $\mathrm{Choo}^{4}$ outlines four types of scanning modes which are framed within two factors that influence scanning: the organisation's perceived analysability of the environment (ie, perceptions of the complexity and rate of change in the environment) and the degree of intrusion into the 
environment (ie, a passive or active approach to information seeking). The four scanning modes are (1) undirected viewing, (2) conditioned viewing, (3) enacting and (4) searching. Undirected viewing and conditioned viewing are non-intrusive, passive forms of information seeking. In undirected viewing, there is an informal approach to information seeking with no specific information needs in mind. Conditioned viewing involves more routine information seeking on specific issues of concern. In contrast, enacting and searching modes involve actively intruding into the environment, and information seeking is focused on to testing the environment (enacting) or formally obtaining objective, quantitative data (eg, surveys) about the environment (searching) to inform decision-making. ${ }^{4}$ Both enacting and searching involve more time and resources than the passive modes of information seeking. ${ }^{4}$

Another study outlines a seven-step process for conducting an environmental scan for a project designed to increase HPV vaccination, ${ }^{31}$ and Longest ${ }^{32}$ proposes an approach to analyse the public policy environment specific to the hospital context. The SWOT (ie, strengths, weaknesses, opportunities, threats) and the STEP, also known as PEST (political, economic, sociocultural and technological), are analytical models that are also used as part of broader business and strategic planning processes for analysis of the internal and external environments, including social and cultural, technological, economic and political/legal environments. ${ }^{21} 2333346465$

Despite the amount of published grey and academic literature that incorporates the environmental scan as a methodological approach, to our knowledge, no reviews have been published on the application of environmental scans within the context of health services delivery. It is not clear which healthcare issues are most commonly addressed; which environments (ie, internal or external) are most often examined; what environmental sectors (eg, technological, social, economic) are most commonly assessed or which conceptual frameworks, if any, are most often used as guides in the scanning process. Although the methodology depends on the research questions and context of the inquiry, it is not known which methods or scanning modes are most often used and for what purposes, and there is limited guidance available in the peer-reviewed or grey literature on designing and implementing environmental scans with regard to structure, selecting data collection methods or analysis techniques. Published reports tend to have very limited, if any, discussion of the limitations of the study. Many papers describe only the methods used, with little to no description of an environmental scan or the rationale for choosing this methodological approach.

Given the resources required for conducting environmental scans and their importance for informing healthcare planning and decision-making, a better understanding of how environmental scans are conceptualised and operationalised in the literature can advance knowledge and may be helpful in guiding practice. A scoping review of this methodological approach may be of particular interest to researchers, policymakers and healthcare professionals who are designing environmental scans to address a particular research question or issue related to health service delivery. A review may also be helpful to healthcare professionals and policymakers who are examining and interpreting evidence from these types of studies to inform policy or practice. This paper describes the protocol for our scoping review that will systematically map the extent, range and nature of evidence examining the use of the environmental scan specifically within the health services delivery context. The scoping review will address the information gaps discussed previously and will explore the application of the environmental scan in the health services delivery literature. The review will include the definitions, characteristics, conceptualisations, theoretical underpinnings, settings, methods, environmental sectors that are most commonly assessed, and the limitations described in these studies. The scoping review will propose a working definition of the environmental scan within the health services delivery context, and it will help lay the groundwork for future research to explore the development of a conceptual framework specific to this field.

\section{METHODS}

Scoping reviews are increasingly used as an effective, rigorous and systematic approach to knowledge synthesis. ${ }^{66-69}$ These types of reviews map key concepts, types of evidence and research gaps related to a particular area that has not been extensively reviewed, and involve systematic searching, selecting and synthesising evidence for the purpose of informing policy and practice ${ }^{69-74}$ Scoping reviews are often undertaken to provide clarity on concepts and working definitions, and to inform future research. ${ }^{71} 727475$ Thus, a scoping review is particularly suitable for addressing the broad research questions for our scoping review that is focused on exploring and increasing understanding of a widely used approach to information seeking but has not yet been comprehensively examined.

Our scoping review will be guided by the evidencebased methodology outlined by Khalil et $a l^{71}$ which is based on the widely recognised frameworks and methodologies of Arksey and O'Malley, ${ }^{74}$ Levac et $a l^{76}$ and the Joanna Briggs Institute. ${ }^{68} 72$ 77-79 The Khalil $e t a l^{71}$ methodology consists of a five-stage approach for conducting scoping reviews based on the work of Levac et $a l^{76}$ : '(1) identifying the research question by clarifying and linking the purpose and question; (2) identifying relevant studies using a three-step literature search to balance feasibility with breadth and comprehensiveness; (3) careful selection of studies using a team approach; (4) extracting and charting the data in a tabular and narrative format and (5) collating the results to identify the implications of the study findings for policy, practice, or research' (pp.119122). ${ }^{71}$ To our knowledge, there are no published scoping 
reviews regarding the use of environmental scans in the context of health services delivery. Our proposed scoping review addresses this research gap. The protocol for the scoping review based on the Khalil et al methodology is described below.

\section{Stage 1: identifying the research questions}

Our main objectives of the scoping review are to (1) map the extent, range and nature of evidence examining the use or application of environmental scans within the health services delivery context; (2) explore definitions, characteristics, conceptualisations, theoretical underpinnings, settings, methods, environmental sectors that are most commonly assessed, study limitations and other features of the environmental scan; (3) propose a working definition for the environmental scan specific to the health services delivery context and (4) identify knowledge gaps and lay the groundwork for future research to explore the development of a conceptual framework in this context. The following broad research questions were identified through an iterative approach with the research team:

1. How have environmental scans been conceptualised and operationalised by researchers, healthcare professionals, policymakers and other stakeholders in the health services delivery research literature? (Objectives 1 and 2)

2. What are the definitions, characteristics and theoretical underpinnings used within environmental scan studies in the context of health services delivery? (Objectives 2 and 3 )

3. What healthcare issues are addressed through the use of environmental scans in the context of health services delivery, including in what settings and for what purposes? (Objectives 2 and 3 )

4. What environments (internal/external) and which environmental sectors are most commonly addressed in environmental scan studies in the context of health services delivery (eg, political, socioeconomic, technological environments)? (Objectives 2 and 3)

5 . What types of study designs and methods have been used in environmental scan studies in the context of health services delivery? (Objectives 2 and 4)

6 . What are the limitations, if any, that are described in the included studies that use environmental scans in the context of health services delivery? (Objective 2 and 4)

For the purposes of this review, the term 'health services delivery' is consistent with WHO's definition ${ }^{80}$ and refers to the direct delivery of health services across the continuum of care including health promotion, disease prevention, diagnosis, treatment, disease management, rehabilitation and palliative care services, across various levels and sites of care within the healthcare system.

\section{Stage 2: identifying relevant studies}

In consultation with the research team, an experienced research librarian (LB) developed a comprehensive three-step search strategy according to established scoping review methodology. ${ }^{71} 727479$ As a measure of rigour, we incorporated a peer review of the search strategy by a second experienced health sciences librarian (KM) according to the Peer Review of Electronic Search Strategies (PRESS) guidelines. ${ }^{81} 82$ To ensure a comprehensive scope of existing evidence on the application of environmental scans in the health services delivery context, we will search both the published and unpublished literature. The term 'health services delivery' is very broad and although it may generate a breath of evidence and reduce the likelihood of missing relevant articles, it can also result in a large and somewhat unmanageable number of references. The team will review the search results on determining the volume and scope of the literature and iteratively decide if changes to the inclusion criteria are needed.

The first step of the search strategy entailed a search of two databases, CINAHL and MEDLINE, to identify titles and abstracts of studies that incorporated the use of environmental scans in the context of health services delivery. At this preliminary stage in the search strategy, we reviewed text words in the titles and abstracts, and articles, and in consultation with the team identified additional keywords and search terms that we will incorporate into the second more comprehensive search of several databases.

We identified the following electronic databases to be searched for relevant papers: CINAHL, MEDLINE, PsycINFO, ERIC, Embase, Canadian Business \& Current Affairs (CBCA) and Academic Search Premier. Box 1 outlines the search strategy. The search strategy is specific for MEDLINE via Ovid and the librarians will assist with translating the search strategies for other listed databases. Once completed, the searches from each database and citations will be imported into Covidence, a web-based platform that organises search results and assists with screening, creating forms and data extraction.

The third step of our search strategy will include a search of the grey literature and a hand-search of the reference lists of all included articles to ensure that all relevant literature is identified. The grey literature search will follow the Canadian Agency for Drugs and Technologies in Health's (CADTH) checklist for searching health-related grey literature. ${ }^{83}$ A Google web search will also be conducted using advanced searching techniques such as file type, in text, synonyms, quotations or all in title. In addition, we will search the New York Academy of Medicine's Grey Literature Report.

\section{Stage 3: selecting studies}

Stage 3 of the scoping review will involve the selection of the studies to be included in the review and will include (1) screening abstracts and titles and (2) full-text screening. 


\section{Box 1 Syntax of search terms}

\section{Search strategy}

1. environment* scan $\$ 4 . t i, a b, k f$.

2. exp 'Delivery of Health Care'/

3. exp Health Services/and (administer* or delegat* or deliver* or distribut* or provide or providing or provision).ti,ab.

4. ((care or healthcare) adj4 (accessib* or availab* or disparit* or equit $^{*}$ or equalit* or inaccessib* or inequalit*)).ti,ab,kf.

5. ((care or healthcare) adj4 (administer* or delegat* or deliver* or distribut* or provide or providing or provision)).ti,ab,kf.

6. ((care or healthcare) adj reform*).ti,ab,kf.

7. case management.ti,ab,kf.

8. (e health or e mental health or ehealth or $m$ health or mhealth or mobile health or telehealth or telemedicine).ti,ab,kf.

9. (health care adj4 (accessib* or availab* or disparit* or equit* or equalit* or inaccessib* or inequalit*)).ti,ab,kf.

10. (health care adj4 (administer* or delegat* or deliver* or distribut* or provide or providing or provision)).ti,ab,kf.

11. (health adj2 reform*).ti,ab,kf.

12. managed care.ti,ab,kf.

13. practice pattern*.ti,ab,kf.

14. prescribing pattern*.ti,ab,kf.

15. ((program* ${ }^{\star}$ or service $\left.{ }^{\star}\right)$ adj4 (accessib* or availab* or disparit* or equit* or equalit* or inaccessib* or inequalit*)).ti,ab,kf.

16. ((program* or service*) adj4 (administer* or delegat* or deliver* or distribut* or provide or providing or provision)).ti,ab,kf.

17. or $/ 2-16$

18. 1 and 17

\section{Inclusion/exclusion criteria}

Our research team developed the following inclusion and exclusion criteria to guide the search strategy and to screen and select studies to be included in the scoping review. To ensure a wide scope of the literature, studies will not be limited to a specific publication date, population, health service, healthcare setting (eg, primary care, acute care), healthcare discipline or geographical location and will be considered for inclusion if they:

- are specific to health services delivery;

- incorporate the use of environmental scans as a methodological approach;

- are published in English or French and

- are (1) primary research studies and (2) grey literature, such as government reports, policy documents and dissertations.

Studies will be excluded if they:

- do not indicate the use of an environmental scan as a methodological approach;

- focus on other healthcare elements, such as professional development and performance management but not specifically on health service delivery and

- are review papers; however, their references lists will be hand-searched for relevant articles to include in the scoping review.

\section{Screening abstracts and titles}

Two reviewers will independently conduct the first level screening of titles and abstracts against the established inclusion and exclusion criteria. We will conduct a pilot test on 50 titles and abstracts to evaluate reviewer agreement in the screening process. Discrepancies in agreement will be resolved through discussion between the reviewers. Adjustments may be made to the inclusion criteria if necessary to ensure consistent interpretation and application of the criteria. The two reviewers will independently screen the remaining abstracts and titles. If discrepancies in agreement related to study selection cannot be resolved after discussion between the reviewers, the decision will be made by a third reviewer.

\section{Screening full text}

Two reviewers will screen the full-text articles independently to determine if they meet the inclusion criteria. We will pilot test 10 full-text articles to assess reviewer agreement. Disagreements will be resolved by the reviewers through discussion or if necessary by a third reviewer. An electronic screening form will be used and the reasons for excluding studies will be documented. A Preferred Reporting Items for Systematic Reviews and Meta-Analysis (PRISMA) flowchart that outlines the search decision process and number of studies included at each phase of the process will be included in the final scoping review. ${ }^{71} 727984$

\section{Stage 4: charting the data}

Working independently, two reviewers will chart (ie, extract) the data using a data abstraction table developed by the research team. The information and study characteristics to be extracted will include title, authorship, year of publication, country(ies) of origin, study purpose, health delivery issue being addressed, study population, setting (eg, primary care, acute care, rehabilitation, home care, long-term care, community), definition of environmental scan, theoretical perspective, environment being assessed (internal/external), environment sectors being assessed (eg, political, technological, social), study design, methods, scanning modes and limitations that are described in these studies. As charting is an iterative process, changes to the data charting table may evolve as we become more familiar with the data and thus ensure that the research questions are addressed. For example, we may add additional categories of data deemed relevant to answer the research questions to the table.

In keeping with established methodology for scoping reviews, we will begin the charting with a pilot study test of 10 articles using the data extraction template to assess consistency between reviewers and to ensure that their approach is aligned with the objectives of the scoping review. ${ }^{70} 76$ If there are inconsistencies, the research team will review, discuss and make changes to the data abstraction template as necessary. ${ }^{70} 76$

\section{Stage 5: collating results}

We will analyse and present the data in tabular format and will include a descriptive numerical summary of the characteristics of the studies as well as a qualitative thematic 
analysis of the results to illustrate key findings and themes using Braun and Clarke's approach. ${ }^{74765}$ The steps in the Braun and Clarke approach include applying initial codes to the data that reflect the content being generated and then collating these codes into potential themes based on patterns of similar codes. ${ }^{85}$ The themes would be checked across the entire data set by our team to refine the name and to generate a clear name for each theme. ${ }^{85}$ The data most amenable to thematic analysis would be theoretical perspectives, environments being assessed, sectors, scanning modes and limitations.

In reporting our results, we will also highlight knowledge gaps and identify implications for policy, practice and research.

\section{Patient and public involvement}

The protocol was developed without public or patient involvement.

\section{Study status}

As of April 2019, we are in stage 3 of the scoping review. We expect that the charting of the data (stage 4) will be completed by October 2019 and the scoping review will be completed by December 2019 .

\section{DISCUSSION}

Although environmental scans have been widely adopted in the healthcare sector to inform decision-making, ${ }^{123137-59}$ there is no consensus on a definition to guide research and practice. This paper presents a protocol for a scoping review that will map the extent, range and nature of evidence examining the use or application of environmental scans within the health services delivery context. The scoping review will increase understanding about the application of environmental scans and contribute to the advancement of research on this methodological approach. We aim to propose a working definition of the concept and the scoping review will inform future research to explore the development of a conceptual framework specific to conducting environmental scans in this context. To our knowledge, no previous scoping reviews have been undertaken to map the evidence examining the use of environmental scans in the health services delivery context. Developing a protocol for our study provides a rigorous structure for the scoping review. A protocol serves to improve the quality and transparency of the research and potentially reduce duplication of research efforts. ${ }^{86} 87$

Considering the widespread use of environmental scans, the potential impact on policy, planning and strategic decision-making in the health system, and the time and resources devoted to planning and conducting environmental scans, this scoping review will be relevant to policymakers, researchers and other stakeholders working within the healthcare realm, particularly those who may consider incorporating an environmental scan as a methodological approach in future work and research aimed at informing healthcare policy or addressing a health service delivery issue. The findings of the scoping review will be disseminated through a peer-reviewed journal and presented at national conferences.

Author affiliations

${ }^{1}$ Adjunct Faculty, Faculty of Nursing, University of Prince Edward Island,

Charlottetown, Prince Edward Island, Canada

${ }^{2}$ Department of Nursing and Health Sciences, University of New Brunswick, Saint John, New Brunswick, Canada

${ }^{3}$ Department of Psychology, Mount Allison University, Sackville, New Brunswick, Canada

${ }^{4}$ Department of Nursing and Health Sciences, University of New Brunswick, Saint John, New Brunswick, Canada

${ }^{5}$ Maritime SPOR SUPPORT Unit, Halifax, Canada

${ }^{6}$ Department of Nursing and Health Sciences, University of New Brunswick, Saint John, New Brunswick, Canada

${ }^{7}$ Robertson Library, University of Prince Edward Island, Charlottetown, Prince Edward Island, Canada

${ }^{8} \mathrm{PhD}$ Student, Interdisciplinary Studies, University of New Brunswick, Saint John, New Brunswick, Canada

${ }^{9}$ Department of Applied Human Sciences, University of Prince Edward Island, Charlottetown, Prince Edward Island, Canada

Contributors All authors contributed to the project including the preparation and editing of the scoping review protocol. PC developed the initial draft of the protocol and after several iterations with significant input from the research team, all team members (PC, SD, RA, DN, AL, KJK, WM) approved the final manuscript that was submitted for publishing. In consultation with the research team, an experienced MLIS Librarian (LB) developed the search strategy for the scoping review and a second librarian conducted a peer review of the search strategy (KM). Two team members will conduct the screening and data extraction and all authors will contribute to the data synthesis and writing of the final scoping review.

Funding This work was supported by the Canadian Institutes of Health Research. Competing interests None declared.

Patient consent for publication Not required.

Ethics approval Ethics approval is not required for this study.

Provenance and peer review Not commissioned; externally peer reviewed.

Open access This is an open access article distributed in accordance with the Creative Commons Attribution Non Commercial (CC BY-NC 4.0) license, which permits others to distribute, remix, adapt, build upon this work non-commercially, and license their derivative works on different terms, provided the original work is properly cited, appropriate credit is given, any changes made indicated, and the use is non-commercial. See: http://creativecommons.org/licenses/by-nc/4.0/.

\section{REFERENCES}

1. Aguilar FJ. Scanning the business environment. New York: Macmillan, 1967.

2. Fahey L, King WR. Environmental scanning for corporate planning. Business Horizons 1977;20:61-71.

3. Jain SC. Environmental scanning in U.S. corporations. Long Range Plann 1984;17:117-28.

4. Choo C. Environmental scanning as information seeking and organizational learning. Information Research 2001;7:1-26.

5. Zhang X, Majid S, Foo S. Environmental scanning: an application of information literacy skills at the workplace. J Inf Sci 2010;36:719-32.

6. Choo C. The art of scanning the environment. Bull Am Soc Inf Sci Technol 1999;25:21-4.

7. Costa J. An empirically-based review of the concept of environmental scanning. Int Journal of Contemporary Hospitality Management 1995;7:4-9.

8. Albright K. Environmental scanning: radar for success. The Information Management Journal 2004:38-44.

9. Abels E. Hot topics: Environmental scanning. Bull Am Soc Inf Sci Technol 2002;28:16-17.

10. Kumar K, Subramanian R, Strandholm K, et al. Competitive strategy, environmental scanning and performance: a context specific 
analysis of their relationship. International Journal of Commerce and Management 2001;11:1-33.

11. Subramanian R, Kumar K, Yauger $C$. The scanning of task environments in hospitals: an empirical study. JABR 1994:10:104-15.

12. Rowel R, Moore ND, Nowrojee S, et al. The utility of the environmental scan for public health practice: lessons from an urban program to increase cancer screening. J Natl Med Assoc 2005;97:527-34.

13. Lozada $\mathrm{H}$, Calantone R. Scanning behavior and environmental variation in the formulation of strategic responses to change. J Bus Ind Mark 1996;11:17-41.

14. Stewart W, May R, Kalia A. Environmental perceptions and scanning in the United States and India: convergence in entrepreneurial information seeking. ET\&P 2008;32:83-103.

15. Duncan RB. Characteristics of organizational environments and perceived environmental uncertainty. Admin Sci Q 1972;17:313-27.

16. Tarver W, Menachemi N. Environmental market factors associated with electronic health record adoption among cancer hospitals. Health Care Manage Rev 2018;43:303-14.

17. Daft RL, Sorumen J, Parks D. Chief executive scanning, environmental characteristics, and company performance. Strategic Manage J 1988;9:123-39.

18. American Hospital Association. The 2018 environmental scan. Trends that are shaping healthcare. H\&HN 2017.

19. Bierbooms J, Bongers I, van Oers H. Strategic market orientation in mental healthcare: a knowledge synthesis. Int $J$ Healthc Manag 2012;5:141-53.

20. Canadian Association of Drugs and Technologies (CADTH). Environmental scan process. Ottawa: CADTH, 2015. https://www. cadth.ca/environmental-scanning. (Accessed Feb 2019).

21. Rodriguez Perera F, Peiro M. Strategic planning in the healthcare sector. Rev Esp Cardiol 2012;65:749-54.

22. Speziale G. Strategic management of a healthcare organization: engagement, behavioural indicators, and clinical performance. European Heart Journal Supplements 2015;17:A3-A7.

23. Tofts A, Walshe K. Managing resources. In: Walshe K. Smith J, ed. Healthcare Management. $2^{\text {nd }}$ ed. New York: Open University Press, McGraw-Hill Education, 2011:452-69.

24. Canadian Association for Drugs and Technology (CADTH). Health priorities in a changing health care landscape. 2018 CADTH Symposium. Ottawa: CADTH, 2018. https://www.cadth.ca/ symposium2018/health-priorities-changing-health-care-landscape. (Accessed Feb 2019).

25. Khan S, Vandermorris A, Shepherd J, et al. Embracing uncertainty, managing complexity: applying complexity thinking principles to transformation efforts in healthcare systems. BMC Health Serv Res 2018;18:192.

26. Kannampallii T, Schauer G, Cohen T, et al. Considering complexity in healthcare systems. J Biomed Inform 2011;44:943-7.

27. Dornan T, McKendree J, Robbe I. Medical education in an age of complexity, uncertainty and reflection. A coda to the Flexner centenary. Med Educ 2011;45:2-6.

28. Smith J, Walshe K. Introduction: The current and future challenges of healthcare management. In: Walshe K, Smith J, eds. Healthcare Management. $2^{\text {nd }}$ ed. New York: Open University Press, McGraw-Hill Education, 2011:1-10.

29. Graham P, Evitts T, Thomas-MacLean R. Environmental scans. How useful are they for primary care research? Can Fam Physician 2008;54:1022-3.

30. Scobba V. PS1-23: The environmental scan: a valuable community health research tool. Clin Med Res 2010;8:184-5.

31. Wilburn A, Vanderpool RC, Knight JR. Environmental scanning as a public health tool: Kentucky's human papillomavirus vaccination project. Prev Chronic Dis 2016;13:E109.

32. Longest $\mathrm{B}$. Management challenges at the intersection of public policy environments and strategic decision making in public hospitals. J Health Hum Ser Adm 2012;35:207-30.

33. Beiko D, Murray E, Davies T, et al. Exploring the business of urology: strategy and strategic planning. Can Urol Assoc J 2018;12:13-17.

34. Hudson R. Marketing situation analysis and strategic planning for NHS clinical services. J Manag Marketing Healthc 2010;3:224-38.

35. Mazurenko O, Menachemi N. Environmental market factors associated with physician career satisfaction. $J$ Healthc Manag 2012;57:307-24.

36. Hsieh HM, Clement DG, Bazzoli GJ. Impacts of market and organizational characteristics on hospital efficiency and uncompensated care. Health Care Manage Rev 2010;35:77-87.

37. Association for Maternal and Child Health Programs. State strategies and initiatives to improve developmental and autism screening and early identification systems. Washington, DC: Association for Maternal and Child Health Programs, 2014. http://www.amchp.
org/programsandtopics/CYSHCN/projects/spharc/Documents/ DevScreeningScan-FINAL-WEB.pdf. (Accessed Feb 2019).

38. DaCosta S, Mukhtar M, Tirillis D, et al. Nurturing the next generation. Early child development service sector review: key findings from the environmental scan. Brampton, Ontario: Region of Peel, 2014. https://www.peelregion.ca/health/nurturing/pdf/NTNG-Early-ChildDevelopment-Service-Sector-Review-Key-Findings-from-theEnvironmental-Scan.pdf. (Accessed Feb 2019).

39. Luke A, Doucet S, Azar R. Paediatric patient navigation models of care in Canada: an environmental scan. Paediatr Child Health 2018;23:e46-e55.

40. Mew EJ, Ritchie D, VanderBurgh D, et al. An environmental scan of emergency response systems and services in remote First Nations communities in Northern Ontario. Int $J$ Circumpolar Health 2017;76:1320208.

41. Rac V, Sahakyan Y, Fan I, et al. The characteristics of stroke units in Ontario: a pan-provincial survey. BMC Health Serv Res 2017;17:154

42. Wijeysundera HC, Trubiani G, Abrahamyan L, et al. Specialized multidisciplinary heart failure clinics in Ontario, Canada: an environmental scan. BMC Health Serv Res 2012;12:236-46.

43. Canadian Mental Health Association. Ontario \& Addictions and Mental Health Ontario. Concurrent disorder services in Ontario: An environmental scan. Toronto, Ontario: Canadian Mental Health Association, 2013. https://ontario.cmha.ca/documents/concurrentdisorder-services-in-ontario-an-environmental-scan/. (Accessed Feb 2019).

44. Moore C, Lee J, Milligan J, et al. Physical activity as medicine among family health teams: an environmental scan of physical activity services in an interdisciplinary primary care setting. Appl Physiol Nutr Metab 2015;40:302-5

45. Naumann D, Reynolds J, McColl M, et al. Environmental scan of programs for fetal alcohol spectrum disorder in Eastern Ontario. Journal of Developmental Disabilities 2013;19:29-49.

46. Gustafson D, Goodyear L, Keough F. When the dragon's awake: a needs assessment of people injecting drugs in a small urban centre. Int J Drug Policy 2008;19:189-94.

47. Porterfield D, Hinnant LM, Kane H, et al. Linkages between clinical practices and community organizations for prevention: a literature review and environmental scan. Am J Public Health 2012;102(Suppl 3):S375-82

48. Aslakson R, Schuster A, Miller J, et al. An environmental scan of advance care planning decision aids for patients undergoing major surgery: a study protocol. Patient 2014;7:207-17.

49. Bednar E, Walsh M, Baker E, et al. Creation and implementation of an environmental scan to assess cancer genetics services at three oncology care settings. J Genet Couns 2018;27:1482-96.

50. Leas B, Berman B, Kash K, et al. Quality measurement in diabetes care. Popul Health Manag 2009;12:265-71.

51. Sibbald SL, McPherson C, Kothari A. Ontario primary care reform and quality improvement activities: an environmental scan. BMC Health Serv Res 2013;13:209-19.

52. Hatch T, Pearson T. Using environmental scans in educational needs assessment. J Contin Educ Health Prof 1998;18:179-84.

53. Hodges B, Albert M, Arweiler D, et al. The future of medical education: a Canadian environmental scan. Med Educ 2011;45:95-106.

54. Maclean R. Resources to address stigma related to sexuality, substance use and sexually transmitted and blood-borne infections. Can Commun Dis Rep 2018;44:62-7.

55. McPherson A, Leo J, Church P, et al. An environmental scan of weight assessment and management practices in paediatric spina bifida clinics across Canada. J Pediatr Rehabil Med 2014;7:207-17.

56. Stacey D, Carley M, Kohli J, et al. Remote symptom support training programs for oncology nurses in Canada: an environmental scan. Can Oncol Nurs J 2014;24:78-88.

57. Baezconde-Garbanati L, Lienemann B, Robles M, et al. Implementation of HPV vaccination guidelines in a diverse population in Los Angeles: results from an environmental scan of local HPV resources and needs. Vaccine 2017;35:4930-5.

58. Blasi P, King D, Henrikson N. HPV vaccine public awareness campaigns: an environmental scan. Health Promot Pract 2015;16:897-905.

59. Jamieson K. An environmental scan of public policy and programs for young Aboriginal children in BC: a cold wind blows. Vancouver, BC: BC Aboriginal Care Society, 2014. https://www.acc-society.bc. $\mathrm{ca} /$ resource/an-environmental-scan-of-public-policy-and-programsfor-young-aboriginal-children-in-bc-a-cold-wind-blows-2/. (accessed Feb 2019).

60. Robinson C, Simmons J. Organising environmental scanning: exploring information source, mode and the impact of firm size. Long Range Planning 2018;51:526-39. 
61. Rathi D, Shiri A, Cockney C. Environmental scan: a methodological framework to initiate digital library development in Canada's north. Aslib Journal of Information Management 2017;69:76-94.

62. Abrahamyan L, Wong W, Pham B, et al. Structure and characteristics of community-based multidisciplinary wound care teams in Ontario: an environmental scan. Wound Rep Reg 2015;23:22-9.

63. Daft RL, Weick KE. Toward a model of organizations as interpretation systems. Academy of Management Review 1984;9:284-95.

64. Public Health Action Support Team. The impact of political economic, socio-cultural, environmental and other external influences. 2017 https://www.healthknowledge.org.uk/publichealth-textbook/organisation-management/5b-understanding-ofs/ assessing-impact-external-influences (Accessed Feb 2019).

65. Ghazinoory S, Mansoureh A, Azadegan-Mehr M. SWOT methodology: a state-of-the-art review for the past, a framework for the future. Journal of Business Economics and Management 2011;12:24-48.

66. Kastner M, Tricco A, Soobiah C, et al. What is the most appropriate knowledge synthesis method to conduct a review? Protocol for a scoping review. BMC Med Res Methodol 2012;12:114.

67. Rumrill P, Fitzgerald S, Merchant W. Using scoping literature reviews as a means of understanding and interpreting existing literature. Work 2010;35:399-404.

68. Tricco A, Lillie E, Zarin W, et al. A scoping review on the conduct and reporting of scoping reviews. BMC Med Res Methodol 2016;16:15.

69. Colquhoun $\mathrm{H}$, Levac D, O'Brien K, et al. Scoping reviews: time for clarity in definition, methods and reporting. J. Clin. Epidemiol 2014;67:1291-4.

70. Daudt H, van Mossel C, Scott S. Enhancing the scoping study methodology: a large, inter-professional team's experience with Arksey and O'Malley's framework. BMC Med Res Methodol 2013;13:48

71. Khalii H, Peters M, Godfrey C, et al. An evidence-based approach to scoping reviews. Worldviews Evid Based Nurs 2016;13:118-23.

72. Peters $\mathrm{M}$, Godfrey $\mathrm{C}$, Khalil $\mathrm{H}$, et al. Guidance for conducting systematic scoping reviews. Int J Evid Based Healthc 2015;13:141-6.

73. Pham M, Rajic A, Greig J, et al. A scoping review of scoping reviews: advancing the approach and enhancing the consistency. Res Synth Methods 2014;5:371-85.

74. Arksey H, O'Malley L. Scoping studies: towards a methodological framework. Int J Soc Res Methodol 2005;8:19-32.
75. Davis K, Drey N, Gould D. What are scoping studies? A review of the nursing literature. Int J Nurs Stud 2009;46:1386-400.

76. Levac D, Colguhoun H, O'Brien K. Scoping studies: advancing the methodology. Implement Sci 2010:5:69.

77. Jordan Z, Lockwood C, Aromataris E, et al. The updated JBI model for evidence-based healthcare. A model reconsidered. The Joanna Briggs Institute 2016 wwwjoannabriggs.org. http://joannabriggs.org/ assets/docs/approach/The_JBI_Model_of_Evidence_-_HealthcareA_Model_Reconsidered.pdf (Accessed Feb 2019).

78. Pearson A, Wiechula R, Court A, et al. The JBI model of evidencebased healthcare. Int J Evid Based Healthc 2005;3:207-15.

79. Aromataris E, Riitano D. Constructing a search strategy and searching for evidence. A guide to the literature search for a systematic review. Am J Nurs 2014;114:49-56.

80. World Health Organization. Health systems service delivery. Geneva: World Health Organization, 2018. http://www.who.int/healthsystems/ topics/delivery/en/. (Accessed Feb 2019).

81. Sampson M, McGowan J, Cogob E, et al. An evidence-based practice guideline for the peer review of electronic search strategies. J Clin Epidemiol 2009;62:944-52.

82. McGowan J, Sampson M, Salzwedeld D, et al. PRESS peer review of electronic search strategies: 2015 guideline statement. J Clin Epidemiol 2016:75:40-6.

83. Canadian Agency for Drugs and Technologies in Health (CADTH). Grey matters: a practical tool for searching health-related grey literature. Ottawa: CADTH, 2018. https://www.cadth.ca/resources/ finding-evidence. (Accessed Apr 2019).

84. Moher D, Liberati A, Tetzlaff J, et al. Preferred reporting items for systematic reviews and meta-analyses: the PRISMA statement. PLoS Med 2009;6:e1000097.

85. Braun V, Clarke V. Using thematic analysis in psychology. Qual Res Psychol 2006;3:77-101.

86. Journal of Medical Internet Research. Why should I publish my protocol or grant proposal? https://jmir.zendesk.com/hc/en-us/ articles/115002860428-Why-should-I-publish-my-protocol-or-grantproposal- (Accessed Feb 2019).

87. University of Toronto Libraries. Systematic \& scoping reviews: methodology behind the search strategies. 2018. What is a protocol? Toronto: University of Toronto Libraries https://guides. library.utoronto.ca/c.php?g=588615\&p=4078120 (Accessed Feb 2019). 